\title{
Environmental enrichment exposure restrains the neuronal damage induced by diabetes and stress in the motor cortex of rat brain
}

\author{
Pamidi N ${ }^{1}$, Satheesha Nayak B ${ }^{2}$, Mohandas Rao $\mathrm{KG}^{2}$, Srinivasa Rao $\mathrm{S}^{2}$, Venu Madhav N ${ }^{2}$ \\ Faculty in Anatomy, Jeffrey Cheah School of Medicine \& Health Sciences, Monash University, Sunway campus, \\ Kuala Lumpur, Malaysia. narendra.pamidi@gmail.com
}

\begin{abstract}
Objective: The present study was intended to assess the effect of environmental enrichment on the diabetes as well as combined actions of diabetes and stress on the neurons of the motor cortex of rat brain. Background: Untreated diabetes mellitus causes severe insults to the neurons of the central nervous system. Treatment with environmental enrichment is known for producing significant and reliable neuronal changes in the neurological disorders of central nervous system.

Materials and methods: Male albino rats of Wistar strain, aged 35 days were used. The rats were divided into (A) Normal Control (B) Vehicle Control (C) Diabetic (D) Diabetes+Stress (E)Diabetes+Environmental enrichment $(F)$ Diabetes+Stress+Environmental enrichment $(n=6)$ in each group). Blood glucose levels and body weight was measured before the induction of diabetes, on the 2 nd day after induction of diabetes and before sacrifice. After exposure to stress and environmental enrichment diabetic rats were sacrificed (Day 30) and brains were processed for cresyl violet staining. The number of survived neurons in the motor cortex was quantified. Results: Quantification of cresyl violet neurons in the motor cortex showed a significant increase in the number of survived neurons in Diabetes+Environmental enrichment and Diabetes+Stress+Environmental enrichment group rats compared to Diabetes and Diabetes+Stress group rats respectively.

Conclusion: Findings from the present study indicated that the exposure to environmental enrichment can prevent the amount of the neural damage caused by complications of diabetes and combined actions of diabetes and stress to the neurons of the motor cortex (Fig. 5, Ref. 37). Text in PDF www.elis.sk. Key words: diabetes, neuropathy, stress, environmental enrichment, motor cortex, neurons.
\end{abstract}

Neuropathy is one of the major complications of diabetes. Severe neuropathy causes thickening of axons, decreased microfilaments and capillary narrowing, which will lead to weakness and unbalanced walking (Tripathi and Srivastava, 2006).Structural and ultra- structural changes in the form of disarrangement of myelin sheath, degenerative changes of neurons and glia, presynaptic vesicle dispersion in swollen axonal bouttons, fragmentation of neurofilaments and oligodendrocyte abnormalities were observed in the cerebral cortex of streptozotocin (STZ) induced diabetic rats (Hernandez-Fonseca et al, 2009).

Motor cortex is a term that describes region of the cerebral cortex involved in the planning, control and execution of voluntary motor functions (Withers and Greenough, 1989, Kleim et al, 1998). Motor cortex is involved in educating to grasp objects precisely, reaching targets more competently and in learning a

${ }^{1}$ Faculty in Anatomy, Jeffrey Cheah School of Medicine \& Health Sciences, Monash University, Sunway campus, Kuala Lumpur, Malaysia, and ${ }^{2}$ Department of Anatomy, Melaka Manipal Medical College, Manipal University, India -516104

Address for correspondence: N. Pamidi, PhD, Faculty in Anatomy, Jeffrey Cheah School of Medicine \& Health Sciences, Monash University, Sunway campus, Kuala Lumpur, Malaysia - 46150.

Phone: +60.1123200734 , Fax: +60.355146001 new movement (Sanes JN, 2003). Motor cortex passes information of planned movement to other brain areas (Cerebellum and basal ganglia) for the proper discharge of the movement.

Exposure to stress causes deleterious effects on body as well as brain. Focusing to stress in life has been revealed to play a significant role in the etiology of various psychiatric illnesses like depression, post-traumatic stress disorder and anxiety (Sapolsky, 1996, Heim et al, 1997, McEwen, 1998, Caspi et al, 2003). Many studies revealed that chronic stress exposure causes alterations in synaptic function and plasticity (Joels M, 2000; Kim JJ and Diamond DM, 2002), dendritic remodeling of medial prefrontal cortex neurons (Brown SM et al, 2005).

Exposure to environmental enrichment (EE) provides an opportunity for stimulating the physical, sensory, social and cognitive activities (Rosenzweig et al, 1962, van Praag et al, 2002). EE exposure is considered as a major therapeutic tool in treating stress and neurodegenerative diseases/disorders. Stimulating environmental conditions significantly influenced the brain functioning by increasing the size and weight of the cortex, neuron sizes and dendritic branching (Mohammed AH et al., 2002). Significant anatomical changes were noticed in cortex as well as sub cortical regions of the brain after EE exposure (Diamond et al, 1976, Kempermann et al, 1997, van Praag et al, 2000). Based on the data in literature, in the present study we aimed to 
understand the effects of EE exposure on diabetes and combined action of diabetes and stress on neurons of rat motor cortex.

\section{Materials and methods}

\section{Animals and groups}

Male albino rats (Wistar strain) 35 days old were used. All animals were maintained in 12:12 hour dark: light environment, in institutional animal house. Animals were given water ad libitum, the standard pellets (Hindustan lever) were used for feeding. Experiment was conducted in compliance with guidelines and principles of the institutional animal ethical committee and the research protocol was permitted by the institutional animal ethical committee for experimental clearance (IAEC/KMC/07/2007-2008).

Rats in the experiment were divided into Normal Control (NC), Vehicle Control (VC), Diabetes(DI), Diabetes+Stress (DI+S), Diabetes + Environmental enrichment $(\mathrm{DI}+\mathrm{E})$, Diabetes + Stress + Environmental enrichment $(\mathrm{DI}+\mathrm{S}+\mathrm{E})$ groups $(\mathrm{n}=6$ in each group). $\mathrm{NC}$ group rats were continued in their home cage without any disturbance and $\mathrm{VC}$ group rats were fed with citric acid buffer solution (pH 4.5) as vehicle.

\section{STZ solution preparation and creating STZ diabetic rat model}

STZ solution of $10 \mathrm{mg} / \mathrm{ml}$ was prepared by dissolving STZ powder (Sigma, St, Louis Mo., USA), in ice-cold citrate buffer ( $\mathrm{pH} 4.5$ ). STZ solution was maintained in cold conditions by keeping it in a tray of ice cubes to prevent decomposition of STZ by temperature. Experimental diabetes was induced with a single intra peritoneal injection of STZ dissolved in sodium citrate buffer ( $\mathrm{pH} 4.5)$ at a dose of $40 \mathrm{mg} / \mathrm{kg}$ body weight and rats were fed with $5 \%$ glucose solution to overcome drug-induced hypoglycemia after STZ injection.

\section{Blood glucose and body weight measurement}

Blood glucose levels and body weight of rats were measured before STZ injection, 2 days after STZ injection and before sacri- fice (day 30). Rat blood was collected from orbital plexus and tail vein and blood sugar was estimated with the 'Accu-Chek Advantage Glucose Monitor'. Rats having blood glucose levels above $225 \mathrm{mg} / \mathrm{dl}$ after the injection of STZ were considered as diabetic and were used for the experiment.

\section{Restraint stress procedure}

The restraint stress paradigm was initiated, after confirmation of diabetes by measuring blood glucose concentrations on the 2nd day of STZ injection. Rats in $\mathrm{DI}+\mathrm{Sand} \mathrm{DI}+\mathrm{S}+\mathrm{E}$ groups were subjected to 6 hrs of daily restraint stress from the 2 nd day of experiment until the end of experiment (Day 30), in a wire mesh restrainer secured with the help of a padlock and latch. A wire mesh restrainer (12 cm length, $5.5 \mathrm{~cm}$ height and $5.5 \mathrm{~cm}$ width) had a wooden base and a stainless steel wire mesh restrainer provided with air holes to prevent overheating attached to the base. Rats were returned to their home cages on completion of the stress session daily and were given access to food before the stress and after the stress sessions. After the final stress session (Day 30), rats were weighed and blood glucose levels were estimated to confirm that diabetic parameters had been maintained in both of the groups.

\section{Enriched environment/ Environmental enrichment}

After confirmation of diabetes by measuring blood glucose levels on the 2 nd day of the experiment, rats in $\mathrm{DI}+\mathrm{E}$ and $\mathrm{DI}+\mathrm{S}+\mathrm{E}$ groups were housed in EE cages daily for 6hoursuntil the end of the experiment (Day 30). The EE was created in a large wood cage (50 cm Length, $50 \mathrm{~cm}$ Width, $29 \mathrm{~cm}$ Height) that contained a variety of objects, including rotating wheels, plastic tubes and toys of different dimensions, which allowed the animals to explore and interact with many different objects. The arrangement and types of objects in the cage varied every day in order to induce novel stimulation of the animals. Animals were placed in the enriched cages in groups of 3-4 rats. When animals were not in the EE, they were housed in standard cages with 1 or 2 other rats.

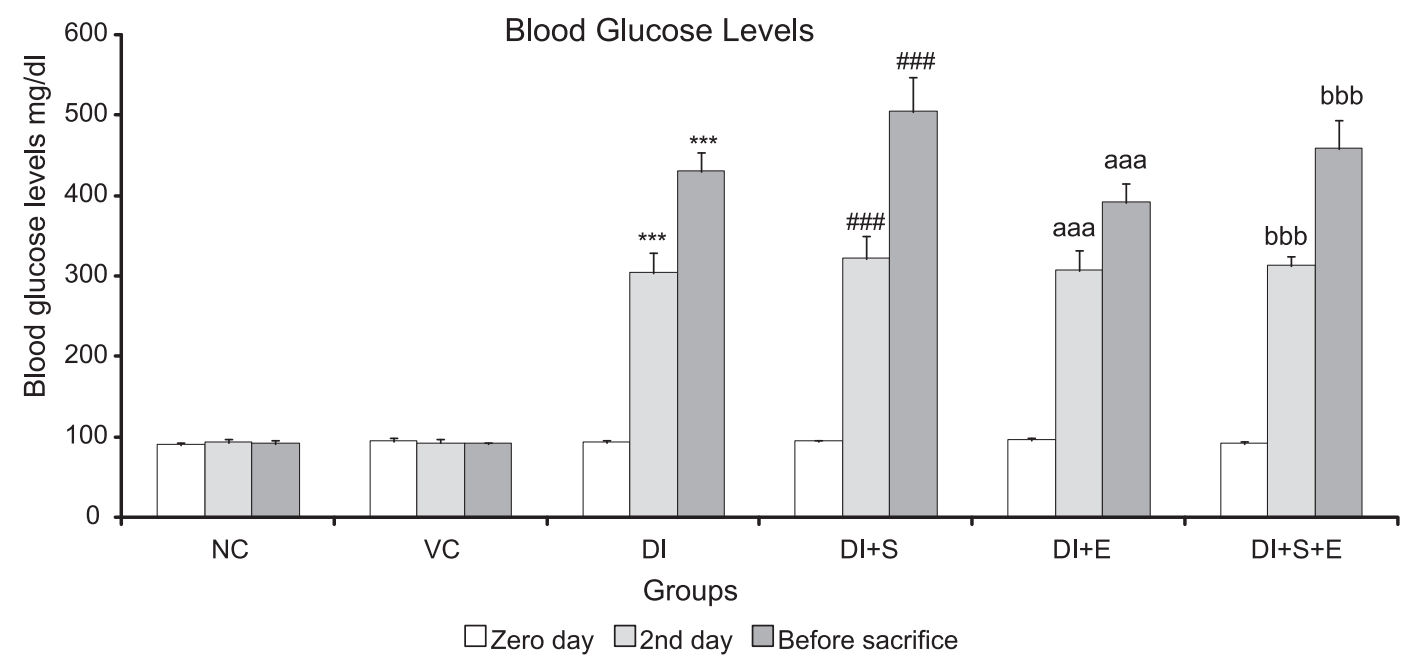

Fig. 1. Bar graph showing the blood glucose levels of rats in different groups. Bars represent mean \pm standard error of mean. NC vs DI: *** p $<0.001$, NC vs DI+S: ${ }^{\# \#} \mathbf{p}<\mathbf{0 . 0 0 1}$, NC vs DI+E: ${ }^{a a a} p<0.001$, NC vs DI+S+E: ${ }^{b b b} p<0.001$. 


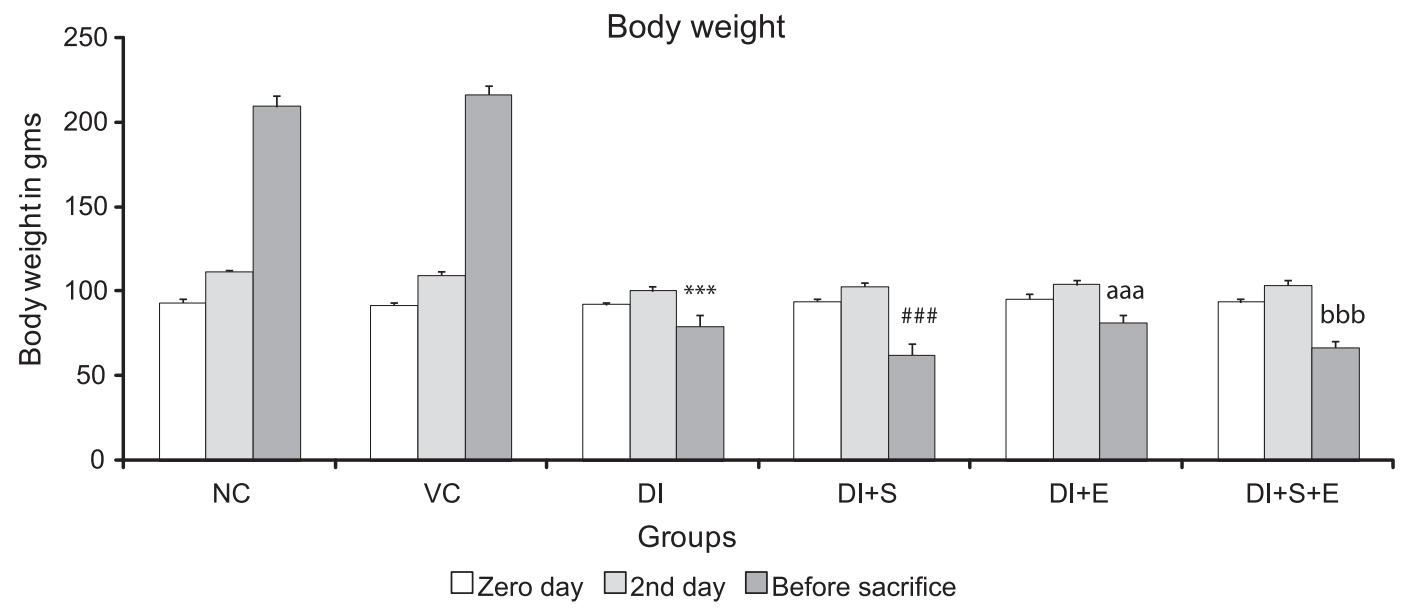

Fig. 2. Bar graph showing the body weight of rats in different groups. Bars represent mean \pm standard error of mean. NC vs DI: *** p<0.001, NC vs DI+S: ${ }^{\# \#} \mathbf{p}<0.001$, NC vs DI+E: aaa $\mathbf{p}<0.001$, NC vs DI+S+E: ${ }^{\text {bbb }} \mathbf{p}<0.001$.

\section{Histological study of Motor cortex}

At the end of the experimental period rats were sacrificed by perfusion method and the brains were post fixed for 48 hours in the Para formaldehyde solution and the tissues were processed through ascending grades of alcohols (50\%-24 hrs, $70 \%-24$ hrs, $90 \%-12 \mathrm{hrs}, 100 \%-12 \mathrm{hrs}$ ) and clearing of alcohol was done with the xylene for about 1-2 hours and paraffin blocks were made in an embedding bath. Coronal sections of $5 \mu$ thickness were cut from the motor cortex using a rotary microtome (Leica RM 2245 , Germany). Twenty five to thirty sections from each animal were mounted serially on air dried gelatinized slides. The sections were stained using $0.01 \%$ cresyl violet stain (Fig. 3).

\section{Neuronal quantification}

Neuronal quantification was done under $40 \mathrm{X}$ of a light microscope (Magnus, Olympus Pvt. Ltd. New Delhi,India). Slides from different groups were coded while counting to avoid manual bias. Ten sections from each rat were selected for cell quantification. The numbers of viable neurons having clear nucleus were quantified. Dark stained neurons with fragmented nuclei, shrunken cell body were excluded from quantification.

\section{Data analysis}

Results were evaluated using analysis of variance (ANOVA) with Bonferroni's multiple comparison test as post hoc test (Graph pad prism 2.01 software). Values are expressed as mean \pm standard error of mean (SEM) and a probability level of $\mathrm{p}<0.05$ was used as the criterion for statistical significance expression.

\section{Results}

\section{Blood glucose levels}

The blood glucose levels of rats in all the groups were similar in the beginning of the experiment $(\mathrm{NC}: 91.03 \pm 2.12, \mathrm{VC}$ : 94.61 \pm 5.19 , DI: $93.5 \pm 2.11$, DI+S: $94.5 \pm 2.37$, DI+E: $95.64 \pm 3.5$, $\mathrm{DI}+\mathrm{S}+\mathrm{E}: 92.17 \pm 2.22$ ) and the blood glucose levels of NC and
VC groups are stable throughout the experimental period. Rats in the experimental groups become diabetic after 2 days following the STZ injection (blood glucose more than $225 \mathrm{mg} / \mathrm{dl}$ ) (DI: 304.7 \pm 24.11 , DI+S: $321.6 \pm 28.26$, DI+E: $306.7 \pm 25.91$, DI+S+E: $312.5 \pm 12.16$ ). The blood glucose levels of rats in the diabetic experimental groups were significantly higher $(p<0.001)$ compared to $\mathrm{NC}$ group from day 2 to till the end of the experimental period (DI: $430.3 \pm 22.89$, DI+S: $505 \pm 42.12$, DI+E: $391.2 \pm 24.01$, DI+S+E: $457.8 \pm 36.17$ ) (Fig. 1).

\section{Body weight}

There was no significant difference among the groups in reference to the body weight in the beginning of the experiment (NC: $93.33 \pm 2.18$, VC: $92 \pm 1.52$, DI: $92.17 \pm 1.16$, DI + S: $94 \pm 1.41$, DI+E: $95.83 \pm 2.94, \mathrm{DI}+\mathrm{S}+\mathrm{E}: 93.67 \pm 1.62)$. There was a reduction in the body weights of rats in diabetic experimental groups on the 2ndday of diabetic period compared to NC group, but this was not significant (NC: $111.8 \pm 1.07, \mathrm{VC}$ : $109.5 \pm 1.97, \mathrm{DI}: 100.3 \pm 2.82$, DI+S: $102.7 \pm 2.58, \mathrm{DI}+\mathrm{E}: 104 \pm 2.75$, DI+S+E: $103.5 \pm 2.95)$ and it was highly significant compared to $\mathrm{NC}$ group before sacrifice $(\mathrm{p}<0.001)$ (NC: $209.5 \pm 6.05, \mathrm{VC}: 216.3 \pm 5.09$, DI: $79 \pm 6.68$, DI+S: $62 \pm 7.29$, DI+E: $81.5 \pm 4.11, \mathrm{DI}+\mathrm{S}+\mathrm{E}: 66.67 \pm 3.88$ ) (Fig. 2).

Effect of EE on the combined actions of diabetes and stress on the numerical cell density of motor cortex (Figs 4, 5).

Microscopic examination of cresyl violet stained sections of rat motor cortex showed significant and reliable changes. Upon quantification of neurons in motor cortex, DI group rats exhibited a significant decrease $(17.79 \pm 0.61, \mathrm{p}<0.001)$ in the mean number of surviving neurons when compared to the NC (31.14 $\pm 0.34)$. Motor cortex of rats in DI+S group also showed a significant decrease $(10.92 \pm 0.36, \mathrm{p}<0.001)$ in the number of surviving neurons when compared to $\mathrm{NC}$ rats(31.14 \pm 0.34$)$. DI+E group rats showed a significant heightening in the number of surviving neurons $(23.57 \pm 0.54, \mathrm{p}<0.001)$ when compared to the DI rats $(17.79 \pm 0.61)$. DI $+\mathrm{S}+\mathrm{E}$ group rats also showed significantly 


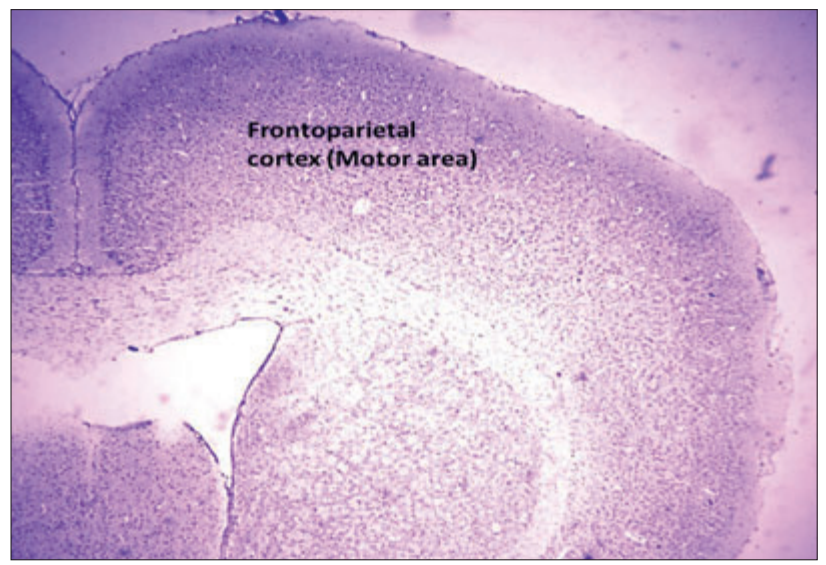

Fig. 3. Photomicrograph showing the motor cortex of rat brain (Cresyl violet stain- $\mathbf{4 X )}$.

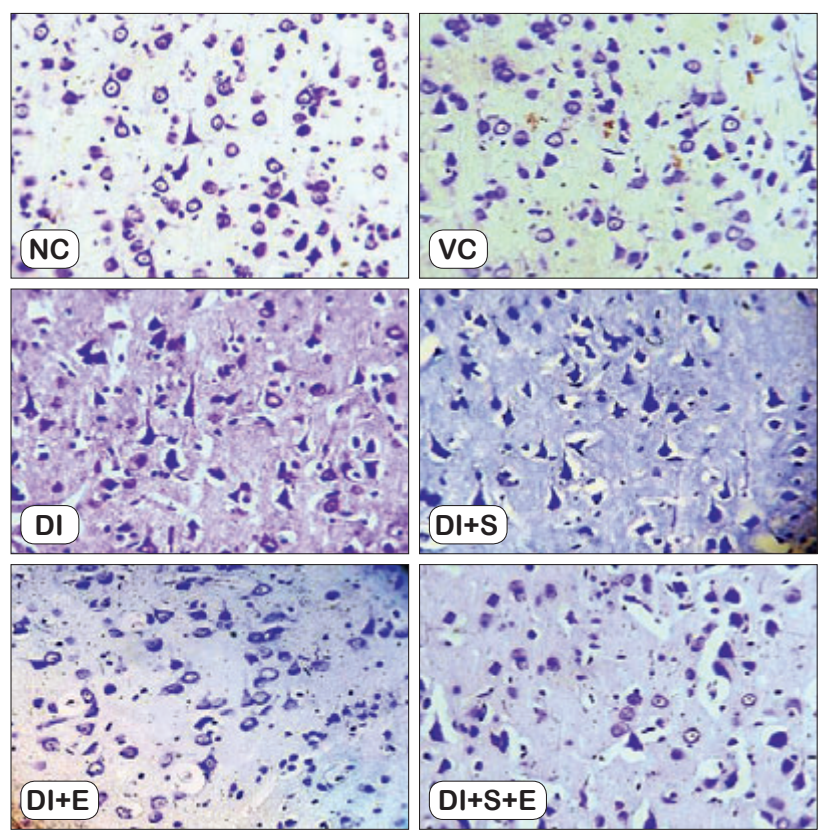

Fig. 4. Photomicrographs of motor cortex (Layer V- 40X, cresyl violet stain) showing the effect of environmental enrichment on diabetes and combined actions of diabetes and stress on the neurons. A significantly increased $(p<0.001)$ number of surviving neurons was observed in $\mathrm{DI}+\mathrm{E}$ group and in $\mathrm{DI}+\mathrm{S}+\mathrm{E}$ group of rats compared to $\mathrm{DI}$ and $\mathrm{DI}+\mathrm{S}$ group of rats, respectively.

$(17.59 \pm 0.48, \mathrm{p}<0.001)$ increased number of surviving neurons in comparison to the DI $+\mathrm{S}$ group rats $(10.92 \pm 0.36)$

\section{Discussion}

A STZ induced experimental diabetic model results in formation of long-lasting oxidative stress in the brain as well as other systems of the body (Kapoor et al, 2009, Xie et al, 2011).Free radicals initiate neuronal death by apoptosis. In addition to the pathological complications of oxidative stress other factors like decreased insulin signaling pathway may also be involved in de- velopment of the diabetic neuropathy and encephalopathy (Dagon et al, 2007). Diabetic complications cause cerebral alterations by abnormal hypothalamic neuropeptide expression, neurotoxicity and changes in glutamate neurotransmission increased circulating corticosterone levels, lack of periodicity, hypersensitivity to stress may contribute to the damage. Morphological alterations and neuronal dysfunctions in the diabetic brain may be also due to reduced level of neurotrophic factors (Wisniewski et al, 2003).

A highly stressful condition of diabetic animals might enhance the vulnerability of different brain areas (Revsin et al, 2005). Previous studies have also shown remodeling (reductions in apical branch number and length) of apical dendrites of pyramidal neurons upon treatment with chronic stress in medial prefrontal cortex neurons (Cook and Wellman, 2004). The process of nerve damage in STZ induced diabetes is similar to nerve damage in human diabetic neuropathy (Reagan and McEwen, 2002). The drug STZ induction into the body causes decreased insulin discharge by destructing the b-cells of the pancreas in the islets of Langerhans. With the help of minute quantity of left over insulin in the pancreas animal can survive for several months but, the disease is fatal without any treatment (Zhang et al, 2008). Highly significant nissl body damage was seen in the motor cortex neurons of rats in $\mathrm{DI}+\mathrm{S}$ group when compared to the $\mathrm{NC}$ rats. The cell bodies of neurons in the DI+S group were shrunken and darkly stained with a degenerative nucleus(Fig 4); this could be due to altered motor cortex volumes as reported by some of the previous studies in the other parts of the brain (McEwen, 2003, McEwen, 2007, McEwen and Milner TA, 2007). Findings from our study indicated that the untreated diabetes mellitus coupled with stress can induce highly significant damage in the central nervous system (CNS) of the rat within 4 weeks of diabetic period or even earlier.

Considering the above degenerative changes in the motor cortex of brain, we treated the animals in DI and DI+S groups with EE. A significant recovery from the damage caused by the diabetes and combined effects of diabetes and stress was observed after treatment with EE. EE exposure brings changes in transcriptional, translational and posttranslational actions which are crucial for neuronal structural modification (Huang et al, 2006). EE expo-

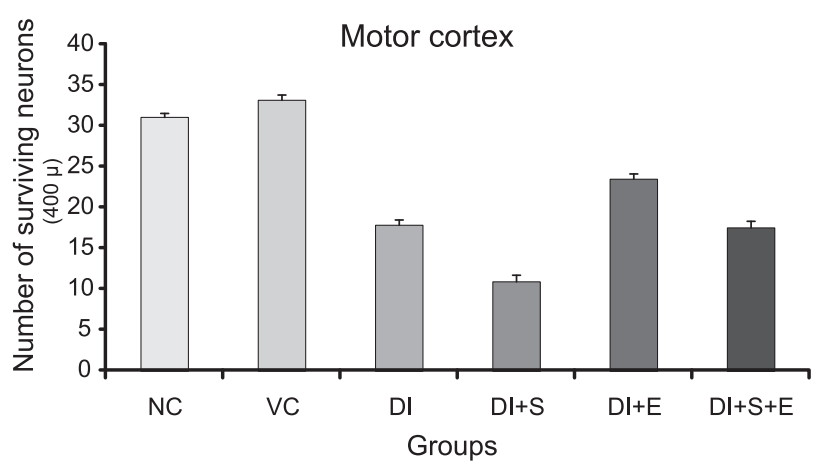

Fig. 5. Bar graph showing the number of survived neurons in the motor cortex region of rat brain. Bars represent mean \pm standard error of mean. NC vs DI: *** p<0.001, NCvs DI+S: ${ }^{\# \#} \mathbf{p}<0.001$, DIvsDI+E: aaa $p<0.001$, DI + S vsDI $+S+E$ : ${ }^{\text {bbb }} p<0.001$. 
sure enhances endothelial cell proliferation in CNS and increases capillary branching and surface area and this activity may lead to improvement in cerebral blood flow. Studies of brain have shown that complex environment enhances synaptic plasticity by mounting long term potentiation, dendritic spine density, expression of synaptic proteins, receptors and neurotrophins (Olsonet al, 2006). EE reduced antidepressive-like effects and elevated levels of serotonin in the prefrontal cortex compared to control and isolation rearing (Brenes et al., 2008).

Treatment with EE significantly increased the dendritic length of pyramidal neurons of auditory (Turner and Lewis, 2003) and parietal cortices (Leggio et al, 2005). In a study by Turner et al $(2002,2003)$ enrichment treatment resulted in high neuronal metabolic activity with more dendritic spines in the motor cortex of mice brain. Rats housed in an enriched environment significantly increased the number of dendritic spines of pyramidal neurons in all the layers of cortex. With the widespread intra and extra cortical connections of pyramidal neurons of cortex may play a role in cortical plasticity (Johansson and Belichenko, 2002).

In the present study, we aimed to observe the protective effect of EE on combined actions of diabetes and restraint stress on rat motor cortex neurons. As explained before, uncontrolled diabetes as well as single housing in restraint stress procedure resulted in highly significant pathological changes in neurons of motor cortex. But upon EE exposure the degeneration of neurons was decreased in DI and DI+S group rats. Within our study, the EE exposure might have brought positive alterations in the levels of neurotrophic factors and neurotransmitters for bringing vigorous changes to prevent the severity of neuronal damage in the DI and DI+S rat brains as explained by Pham TM et al (1999) and the data in the study may designate a new approach for the prevention of some of the CNS complications correlating with diabetes and stress.

\section{Conclusions}

To our knowledge, this is the first study explaining the beneficial effects of EE on combined effects of diabetes and chronic restraint stress on motor cortex neurons. Findings from the present study showed that exposure to EE can prevent the amount of the neural damage caused by complications of diabetes and combined actions of diabetes and stress on the neurons of the motor cortex. The data obtained in the rat motor cortex may be useful to interpret the functional deficits of diabetic patients and for better management of diabetes.

\section{References}

1. Tripathi BK, Srivastava AK. Diabetes Mellitus: Complications and Therapeutics. Med Sci Monit 2006; 12 (7): 130-147.

2. Hernandez-Fonseca JP, Rincon J, Pedreanez A et al. Structural and ultra structural analysis of cerebral cortex, cerebellum, and hypothalamus from diabetic rats. Exp Diabetes Res 2009; 2009: 329-632.

3. Withers GS, Greenough WT. Reach training selectively alters dendritic branching in subpopulations of layer II-III pyramids in rat motorsomatosensory forelimb cortex. Neuropsychologia 1989; 27 (1): 61-69.
4. Kleim JA, Barbay S, Nudo RJ. Functional reorganization of the rat motor cortex following motor skill learning. J Neurophysiol 1998; 80 (6): 3321-3325.

5. Sanes JN. Neocortical mechanisms in motor learning. Curr Opin Neurobiol 2003, 13: 225-231.

6. Sapolsky RM. Why stress is bad for your brain. Science 1996; 273 (5276): 749-570.

7. Heim C, Owens MJ, Plotsky PM, Nemeroff CB. The role of early adverse life events in the etiology of depression and posttraumatic stress disorder. Focus on corticotropin-releasing factor. Ann N Y AcadSci 1997; 821: 194-207.

8. McEwen BS. Protective and damaging effects of stress mediators. N Engl J Med 1998; 338 (3): 171-179.

9. Caspi A, Sugden K, Moffitt TE et al. Influence of life stress on depression: moderation by a polymorphism in the 5-HTT gene. Science 2003; 301 (5631): 386-389.

10. Joels M. Modulatory actions of steroid hormones and neuropeptides on electrical activity in brain. Eur J Pharmacol 2000; 405 (1-3): 207-216.

11. Kim JJ, Diamond DM.The stressed hippocampus, synaptic plasticity and lost memories. Nat Rev Neurosci 2002; 3 (6): 453-462.

12. Brown SM, Henning S, Wellman CL. Mild, short-term stress alters dendritic morphology in rat medial prefrontal cortex. Cereb Cortex 2005; 15 (11): 1714-1722.

13. Rosenzweig MR, Krech D, Bennett EL, Diamond MC. Effects of environmental complexity and training on brain chemistry and anatomy: a replication and extension. J Comp Physiol Psychol 1962; 55: 429-437.

14. vanPraag H, Schinder AF, Christie BR, Toni N, Palmer TD, Gage FH. Functional neurogenesis in the adult hippocampus. Nature 2002; 415 : 1030-1034.

15. Mohammed AH, Zhu SW, Darmopil S et al. Environmental enrichment and the brain. Prog Brain Res 2002; 138: 109-133.

16. Diamond MC, Ingham CA, Johnson RE, Bennett EL, Rosenzweig MR. Effects of environment on morphology of rat cerebral cortex and hippocampus. J Neurobiol 1976; 7 (1): 75-85.

17. Kempermann G, Kuhn HG, Gage FH. More hippocampal neurons in adult mice living in an enriched environment. Nature 1997; 386 (6624): $493-495$.

18. vanPraag H, Kempermann G, Gage FH. Neural consequences of environmental enrichment. Nat Rev Neurosci 2000; 1 (3): 191-198.

19. Kapoor R, Srivastava S, Kakkar P. Bacopamonnieri modulated antioxidant responses in brain and kidney of diabetic rats. Environmental Toxicology and Pharmacology 2009; 27: 62-69.

20. Xie N, Zhang W, Li J et al. $\alpha$-Linolenic Acid Intake Attenuates Myocardial Ischemia/Reperfusion Injury through Anti-inflammatory and Antioxidative Stress Effects in Diabetic But Not Normal Rats. Arch Med Res 2011; 42 (3): 171-181.

21. Dagon Y, Avraham Y, Link G, Zolotarev O, Mechoulam R, Berry EM. The synthetic cannabinoid HU-210 attenuates neural damage in diabetic mice and hyperglycemic heochromocytoma PC12 cells. Neurobiology of Disease 2007; 27 (2): 174-181.

22. Wisniewski K, Fedosiewicz-Wasiluk M, Holy ZZ, Car H, Grzeda E. Influence of NMDA, a potent agonist of glutamate receptors, on behavioral activity in 4-week streptozotocin-induced diabetic rats, Pol J Pharmacol 2003; 55 (3): 345-351. 
197-202

23. Revsin Y, Saravia F, Roig $\mathbf{P}$ et al.Neuronal and astroglial alterations in the hippocampus of a mouse model for type 1 diabetes. Brain Res 2005; 1038 (1): 22-31.

24. Cook SC, Wellman CL. Chronic stress alters dendritic morphology in rat medial prefrontal cortex. J Neurobiol 2004; 60: 236-248.

25. Reagan LP, McEwen BS. Diabetes, but not stress, reduces neuronal nitric oxide synthase expression in rat hippocampus: implications for hippocampal synaptic plasticity. Neuroreport 2002; 13 (14): 1801-1804.

26. Zhang WJ, Tan YF, Yue JT, Vranic M, Wojtowicz JM. Impairment of hippocampal neurogenesis in streptozotocin-treated diabetic rats. Acta Neurol Scand 2008, 117 (3): 205-210.

27. McEwen BS. Mood disorders and allostatic load. Biol Psychiat 2003; 54: 200-207.

28. McEwen BS. Physiology and neurobiology of stress and adaptation: Central role of the brain. Physiol Rev 2007; 87: 873-904.

29. McEwen BS, Milner TA. Hippocampal formation: Shedding light on the influence of sex and stress on the brain. Brain Res Rev 2007; 55: 343-355.

30. Huang FL, Huang KP, Wu J, Boucheron C. Environmental enrichment enhances neurogranin expression and hippocampal learning and memory but fails to rescue the impairments of neurogran in null mutant mice. J Neurosci 2006; 26 (23): 6230-6237.

31. Olson AK, Eadie BD, Ernst C, Christie BR. Environmental enrichment and voluntary exercise massively increase neurogenesis in the adult hippocampus via dissociable pathways. Hippocampus 2006; 16 (3): $250-260$.
32. Brenes JC, Rodríguez O, Fornaguera J. Differential effect of environment enrichment and social isolation on depressive-like behavior, spontaneous activity and serotonin and norepinephrine concentration in prefrontal cortex and ventral striatum. Pharmacol Biochem Behav 2008; 89 (1): 85-93.

33. Turner CA, Lewis MH, King MA. Environmental enrichment: effects on stereotyped behavior and dendritic morphology. Dev Psychobiol 2003; 43 (1): 20-27.

34. Leggio MG, Mandolesi L, Federico F et al. Environmental enrichment promotes improved spatial abilities and enhanced dendritic growth in the rat. Behav Brain Res 2005; 163 (1): 78-90.

35. Turner CA, Yang MC, Lewis MH. Environmental enrichment: effects on stereotyped behavior and regional neuronal metabolic activity. Brain Res 2002; 938 (1-2): 15-21.

36. Johansson BB, Belichenko PV. Neuronal Plasticity and Dendritic Spines: Effect of Environmental Enrichment on Intact and Postischemic Rat Brain. J Cereb Blood Flow Metab 2002; 22 (1): 89-96.

37. Pham TM, Ickes B, Albeck D, Soderstrom S, Granholm AC, Mohammed $\mathbf{A H}$. Changes in brain nerve growth factor levels and nerve growth factor receptors in ats exposed to environmental enrichment for one year. Neuroscience 1999; 94 (1): 279-286.

Received November 5, 2012. Accepted October 27, 2013. 